\title{
Primary Discussion for Enterprise Network Training in M Times
}

\author{
Xuelian $\mathrm{Hou}^{1, \mathrm{a}^{*}}$, Xiangqian Wang ${ }^{1, \mathrm{~b}}$ and Qiang Luo ${ }^{1, \mathrm{c}}$ \\ ${ }^{1}$ State Grid of China Technology College Shandong Tai'an China \\ aLotus0617@163.com, b2v2999122@126.com, ㄷddzdjzx@sina.com.cn
}

Keywords: Enterprise training; Training course; Mobility; Gaming; Personalized

\begin{abstract}
This paper analyzes the necessity of enterprise training, through the discussion to the learning styles and characteristics in net-era, designed the enterprise network training course in the knowledge structure of "1+2+1", and the courseware of " $5 \pm 2$ " model of knowledge point number. Based on the above structure model of training content, it determines the study way gradually to the mobility, gaming, and personalized direction, and it can effectively encourage learning into the subconscious mind to get the best training and learning effect. This structure model can be used for enterprise network training courses.
\end{abstract}

\section{Introduction}

As the promotion of "a strong three-excellent" construction goal, the enterprise staff's training efforts are also greatly growing in state grid. New employees injected fresh blood for the enterprise, new employees training for reserve a large amount of manpower, intellectual resources for the long-term development of the enterprise. In the age of the mobile Internet, enterprise training also gradually shift to the direction of the network training.

\section{Necessity of Enterprise Training}

From the perspective of human resource (HR), enterprise operation can't work without people. Throughout the human resources work, there are two main sources of human way.

The Campus Recruitment. To the population who will leave campus and come to the society, they are not familiar with the work environment and working content, for the prepared people they should be have a certain understanding, that is "know", but they may not know how to do, in other words they are lack of certain working experience. If you want to better in the industry, or better based on the industry, they need enough time to familiar with and learn the related knowledge.

Social Recruitment. For professional who already has the work experience, they have enough understanding of industry knowledge, and also be able to accomplish a certain number of things, to work and can mount guard is their advantage. However, because they have work experience before, maybe they can't agree or agree all the new enterprise's concept, the way they do also do not comply with the standards of this enterprise, so this part of the human resources also have a certain deficiencies Based on the above two ways of enterprises to achieve high growth, there can be the third source of human.

Cultivate My Own Business. According to the demand of human resources of enterprise, combining with the actual needs of specific work, the targeted training for the employees make the staff not only know the industry knowledge and industry culture, also can have a higher sense of identity of the enterprise concept, and the employees will do things in accordance with the enterprise standard in the work. This is the fundamental reason of do corporate training.

In recent years, the national grid on the new employees training has been sticking to "first training, then induction" mode, new employees training work is becoming more and more refined, and the new staff has more profound, more accurate understanding to the national grid[1]. One of the national grid human sources is college graduates; In order to make the new employees can put theoretical knowledge into practical ability as soon as possible, system training about enterprise culture, professional knowledge is necessary[2]. 


\section{Learning Style and Characteristics of the M Times}

Peter Senge once said. the only lasting competitive advantage, is having the ability of learning faster than its competitors[3]. We want to learn, because we "existence crisis", we can form acquire knowledge, skills, and achieve the goal of improve personal ability from learning. However, different learning motivation, the way of learning, experience learning process, and learning after the application is different, all these will decide the different learning effect.

Based on the mobile Internet era of learning, mainly has the following several ways:

Embedded Learning. To embed the learning to workflow, to learn and play. The feature of this way is the learning process is short, and study effect is most obvious. By this way, the employee does not affect work, and finish goal-oriented learning. Also by learning to better optimize the ability to work, it is in line with the adult learning characteristics of "to learn to play" and targeted learning.

Fragmented Learning. Under the current mobile Internet era, people's learning turns into the open, diverse style, learning gradually presents the fragmentation. At the same time, mobile Internet technology makes learning environment and learning cognitive present fragmentation, Fragmentation learning are a big shift of learning style[4]. Effective use of fragmented time, undertake personalized learning, will be accumulated into logical pieces of knowledge. Mobile learning provides us with the learning anytime and anywhere, in spare time to do separated learn, and it constitute the characteristic of fragmentation, at the same time, this way can better improve the absorption of knowledges [5].

Social Learning. With the support of various APP, through different mobile terminals, learning go towards the social. The most important feature is human-centered interaction, more of fragmented learning with organized and formal of informal learning.

According to Maslow's needs hierarchy chart, Training to be successful, we should adhere to the people-oriented and well the human nature. Only respect, trust and success staffs first, the following is achievement enterprise's development. The fundamental driving force of Organization's development is learning and development, and in the learning process we should respect for humanity, and inspire staff's ability development. In the mobile Internet era, work, study and life has been unable to separate, Enterprises should also actively transform, to make employees learn from passive learning into active learning, realize the integration of learning and work, autonomous learning in ubiquitous.

\section{The Content Planning of the Enterprise Training Course}

Enterprises training want to develop in the competition and innovation, it is necessary to build the corresponding course system, matched channels and operation mode. In the era of mobile Internet, in the enterprise training we should give priority to content, in the content planning we should pay attention to the knowledge structure and the number of knowledge points.

Knowledge Structure of “ $\mathbf{1 + 2 + 1}$ ". According to the survey, on a problem, people can't pay attention on it more than 4 minutes. In training courseware, we'd better to set about 4 minutes for a knowledge point, and the structure is " $1+2+1 "$.

Table 1 " $1+2+1$ ” knowledge structure

\begin{tabular}{|c|c|c|c|}
\hline Teaching link & introduce & explain & summarize or review \\
\hline Times & 1 minute & 2 minutes & 1 minute \\
\hline
\end{tabular}

The Courseware Knowledge Points Number of " $5 \pm 2$ ". Based on analysis of Duan Xiaoju in her paper named "Development of working memory span about eight to adulthood", person's memory span is change, the maximum capacity of the memory can't be more than 7[6]. People's short-term memory ability is limited, usually short-term memory of knowledge points in " $5 \pm 2$ " (3-7) range, So in the training course a courseware set 3-7 knowledge points is advisable. 


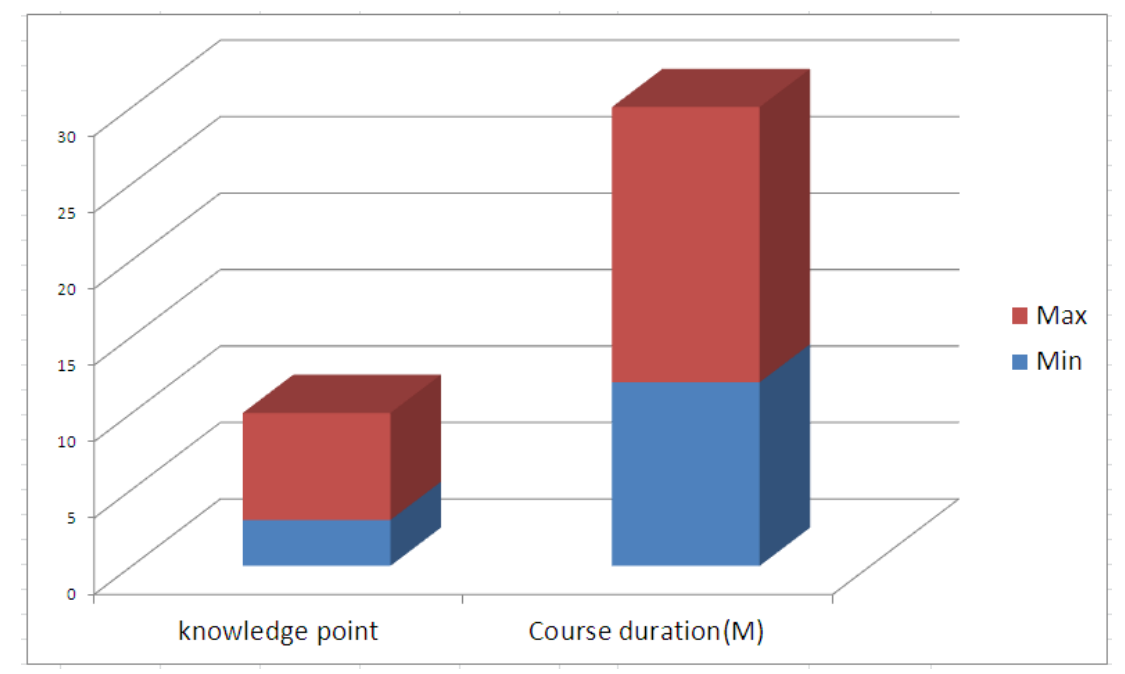

Figure 1. The relationship of knowledge point and course duration

\section{The Future of Enterprise Training in M Times}

Successful College Online should be a platform of knowledge precipitation, interaction and communication, and course completion, course selection rate and the student login rate are rational index to evaluate whether the college online successful or not. In M times, network training will be mobility, gaming, and personalized.

Mobility. With the rapid development of mobile terminal and the support of network communication technology, learning style turns from the traditional classroom learning into mobile learning, its biggest characteristic is the random learning anytime and anywhere.

Gaming or contextualized. With the support of mobile learning and the development on all kinds of mobile APP, Gaming or contextualized way to learn attract the learners more and more, it greatly inspired the learners' interest in learning, and it also can achieve well learning effect.

Personalized. On the way to learn, from "everyone is learning" to "at any time to learn", and then to the current "Everywhere can learn", People are more focused on learning the degrees of freedom, flexibility and personalization. Mobile learning based on mobile terminals can reflect learning more personalized, according to individual needs, learners set up a personalized learning solutions and plans.

Hybrid learning. At present, there are four platforms for Mobile terminal, Smart phone, PAD, computer and television. Based on the current cloud platform, with the internet of things, we can realize the selection of hybrid learning platform.

\section{Summary}

The ultimate purpose of learning is to make learning into the subconscious mind; learning should to be around the human nature; because under the natural state, learning effect will be the best. When learning becomes a subconscious behavior, it means you are used to study and learn knowledge. How to do unified strategy understanding, and how to unified working language and behavior, including new unified form, professional skills, management skills and so on, it is worth for enterprise training as its network training mission.

\section{References}

[1] Caiyun Guo, Xinan He, Qitao Song. The analysis and discussion about new employees training of State Grid Corporation of China[J].State Grid Technical College academic journal,2014,17(1):63-67. 
[2] Huaqing Yue. Thinking and suggestion of new staff training in State Grid Corporation[J]. State Grid Technical College academic journal, 2014,17(3):59-61.

[3] Jianmin Zhao. The university core competitiveness theory resource and essential reading[J]. China Adult Education, 2008, 6:5-6.

[4] Mi Wang. The design of micro video content oriented fragmentation learning age[D]. East China Normal University (ECNU), 2013.

[5] Ying Shan. Fragmentation and entertainment learning based on MOOC and Micro course[J]. The Chinese Journal of ICT in Education, 2014, 19(1):18-19.

[6] Xiaoju Duan. Development of working memory span about eight to adulthood[J] , Psychological science,2009,32(2):324-326.

[7] Zhi-hui Xiao. The Reviewed Mobile Internet research[[J]. Telecommunications Science, 2009(10):30-36.

[8] Xiao-hui $\mathrm{Yu}$. The development and thinking of mobile Internet [J]. Telecommunications Network Technology,2008(12):21-27.

[9] Wen-tao Kang. The research of mobile Internet's development trend[J/OL]. Electronic manufacture, 2015(05).http://www.cnki.net/kcms/detail/11.3571.TN.20150428.1411.116.html.

[10]Clive Thompson. How Khan Academy Is Changing the Rules of Education[DB/OL].http://www.wired.com/magazine/2011/07/ff_khan/,2011-07-10. 